\title{
Convergence of vitamin D and lysophosphatidic acid signaling in stimulating human osteoblast maturation
}

\begin{abstract}
Jason P. Mansell *
Department of Biological, Biomedical and Analytical Sciences, University of the West of England, Bristol, UK

${ }^{*}$ Correspondence: jason.mansell@uwe.ac.uk
\end{abstract}

Edited and reviewed by:

Ovidiu Constantin Baltatu, University Camilo Castelo Branco, Brazil

Keywords: vitamin D, lysophosphatidic acid, osteoblast, alkaline phosphatase, differentiation

\section{A commentary on}

Vitamin D and gene networks in human osteoblasts

by van de Peppel, J., and van Leeuwen, J. P. T. M. (2014). Front. Physiol. 5:137. doi: 10.3389/fphys.2014.00137

Vitamin D is absolutely essential for the provision of a mechanically robust, mineralized bone collagen matrix. Whilst their continues to be a debate over the precise role of this steroid in bone biology it is unequivocal that active vitamin D3 metabolites have direct effects on the control of human osteoblast (hOB) function, matrix calcification and overall bone metabolism. Indeed van de Peppel and van Leeuwen identify many studies supporting a direct effect of the active vitamin D3 metabolite, 1,25-dihydroxyvitamin D3 $(1,25 \mathrm{D})$, in hOBs. It is also clear that these cells are receptive to $24,25 \mathrm{D}$, and that the responses observed are a consequence of vitamin D receptor (VDR)-mediated events. Of additional significance are the reports describing cross-talk between $1,25 \mathrm{D}$ and other growth factors known to influence hOB activity. These are especially important developments that will help decipher the multiple networks which interconnect with vitamin D in controlling gene expression linked to the regulation of cell growth, bone matrix synthesis and mineralization. The identities of these molecules in turn offer enticing opportunities in developing novel strategies to facilitate $1,25 \mathrm{D}$ enhanced bone repair and/or regeneration where it might be needed most, for example at sites of fracture non-unions and at bone biomaterial surfaces to bolster their integration into host tissue.
The cited growth factors known to participate with $1,25 \mathrm{D}$ are wide ranging and include local bone mediators, e.g., TGFbeta, the calciotropic hormone PTH and Wnt signaling ligands. Collectively these studies underpin the diversity of signal transduction networks that converge on $1,25 \mathrm{D}$ in regulating hOB function. There is however a surprising omission by van de Peppel and van Leeuwen; the simple bioactive glycerophospholipid, lysophosphatidic acid (LPA). This particular lipid is one of only three reported growth factors known to synergistically co-operate with $1,25 \mathrm{D}$ in promoting hOB maturation; TGFbeta and EGF are the other two agents. Approximately a decade ago Mansell's group (Gidley et al., 2006) discovered that the ability of $1,25 \mathrm{D}$ to stimulate hOB (MG63) alkaline phosphatase (ALP) expression was a consequence of signaling co-operation with serum-borne LPA, as bound to the albumin fraction. In the absence of serum 1,25D had, at best, a trivial influence on ALP expression. Since this initial finding several published works have emerged describing the co-operative effects of LPA and selected LPA receptor agonists with $1,25 \mathrm{D} / 24,25 \mathrm{D}$ on $\mathrm{hOB}$ formation and maturation, not only in a conventional tissue culture setting but in association with titanium and hydroxyapatite, two widely used bone biomaterials (Blackburn and Mansell, 2012; Lancaster et al., 2014).

It has been known for over two decades that the bulk of serum LPA, the most abundant growth factor in serum, is stably bound to the albumin fraction. Indeed an explanation as to why serum starved fibroblasts accrued enormous quantities of F-actin stress fibers in response to serum was attributed to albumin bound
LPA (Ridley and Hall, 1992). Similarly Tigyi and colleagues discovered that the serum-borne component responsible for evoking membrane currents in Xenopus oocytes and neurite retraction in $\mathrm{PC} 12$ pheochromocytoma cells was LPA tethered to albumin (Tigyi, 2010). Given that $1,25 \mathrm{D}$ co-operates with LPA in promoting hOB maturation this raises a potentially important point pertaining to studies investigating the in vitro effects of $1,25 \mathrm{D}$, either alone, or in combination with selected agents. It is likely that such studies are inadvertently reporting on signaling cross-talk with LPA associated with whole serum or native, serum albumin. Greater attention should therefore be placed in defining the culture conditions when examining the influence of $1,25 \mathrm{D}$ on hOBs; essentially fatty acid-free albumin(s) or charcoal stripped sera are obvious alternatives to eliminate signaling interference from LPA.

The ability of $1,25 \mathrm{D}$ to act in concert with LPA in promoting ALP expression is an exciting development in hOB biology. Although it is generally agreed that $1,25 \mathrm{D}$ influences the expression of hOB genes synonymous with their transition to a more mature phenotype, the role of $1,25 \mathrm{D}$ in the context of ALP expression has yet to be more clearly defined. Despite the clear connection between 1,25D and bone mineralization and the vital role of ALP in this process, ALP, unlike osteocalcin for example, is not identified as a $1,25 \mathrm{D}$ responsive gene. This is a particularly intriguing paradox. Whilst there is evidence of a vitamin D response element like-motif in the promoter region of the human ALP gene it is reportedly non-functional. It has been proposed that a role of $1,25 \mathrm{D}$ in ALP expression is a 
consequence of enhanced mRNA stability rather than direct transcriptional control (Orimo and Shimada, 2006; Orimo, 2010). In this regard $1,25 \mathrm{D}$ amplifies protein expression by extending the life of the ALP transcript. It is conceivable therefore that the synergistic increases observed for hOB ALP consequent to co-stimulation with $1,25 \mathrm{D}$ and LPA, is an enhancement, of an otherwise modest, LPA-induced event. Credence is given to this possibility because LPA (likewise for TGFbeta and EGF), when administered to hOBs, in isolation, stimulates a small increase in ALP (Blackburn and Mansell, 2012). In accordance to the remarks of van de Peppel and van Leeuwen research could now extend to identifying the signaling networks that exist for LPA and 1,25D in hOBs whether they are related directly to hOB fate or to other cell types within the bone microenvironment.

The discovery of signaling co-operation between LPA and 1,25D in the regulation of ALP is an important development given the vital role of this enzyme in bone matrix calcification (Whyte, 2010). Importantly our findings echo an earlier, very significant event in our understanding of signal transduction "cross-coupling" wherein a promitogenic stimulus (e.g., LPA) converges with a pro-differentiating steroid hormone (e.g., 1,25D) response (Schule and Evans, 1991). Identifying LPA as a molecule with the capacity to enhance 1,25D-induced ALP adds another interest- ing layer that may extend to the processes of bone repair and regeneration; LPA is a product of platelet activation and a recognized mediator of soft tissue healing (Moolenaar et al., 2004). There is potential therefore to consider LPA and related molecules in a bone regenerative context, either in a controlled release format or as coatings to contemporary metal and ceramic devices.

\section{REFERENCES}

Blackburn, J., and Mansell, J. P. (2012). The emerging role of lysophosphatidic acid (LPA) in skeletal biology. Bone 50, 756-762. doi: 10.1016/j.bone.2011.12.002

Gidley, J., Openshaw, S., Pring, E. T., Sale, S., and Mansell, J. P. (2006). Lysophosphatidic acid cooperates with $1 \mathrm{a}, 25(\mathrm{OH}) 2 \mathrm{D} 3$ in stimulating human MG63 osteoblast maturation. Prostaglandins Other Lipid Mediat. 80, 46-61. doi: 10.1016/j.prostaglandins.2006.04.001

Lancaster, S. T., Blackburn, J., Blom, A., Makishima, M., Ishizawa, M., and Mansell, J. P. (2014). 24,25-Dihydroxyvitamin D3 cooperates with a stable, fluoromethylene LPA receptor agonist to secure human (MG63) osteoblast maturation. Steroids 83, 52-61. doi: 10.1016/j.steroids.2014. 01.010

Moolenaar, W. H., Van Meeteren, L. A., and Giepmans, B. N. G. (2004). The ins and outs of lysophosphatidic acid signaling. BioEssays 26, 870-881. doi: 10.1002/bies.20081

Orimo, H. (2010). The mechanism of mineralisation and the role of alkaline phosphatase in health and disease. J. Nippon Med. Sch. 77, 4-12. doi: 10.1272/jnms.77.4

Orimo, H., and Shimada, T. (2006). Posttranscriptional modulation of the human tissue-nonspecific alkaline phosphatase gene expression by 1,25-dihydroxyvitamin D3 in
MG-63osteoblastic osteosarcoma cells. Nut. Res. 26, 227-234. doi: 10.1016/j.nutres.2006. 05.004

Ridley, A. J., and Hall, A. (1992). The small GTPbinding protein Rho regulates the assembly of focal adhesions and actin stress fibers in response to growth factors. Cell 70, 389-399. doi: 10.1016/ 0092-8674(92)90163-7

Schule, R., and Evans, R. M. (1991). Cross-coupling of signal transduction pathways: zinc finger meets leucine zipper. Trends. Genet. 7, 377-381. doi: 10. 1016/0168-9525(91)90259-S

Tigyi, G. (2010). Aiming drug discovery at lysophosphatidic acid targets. Br. J. Pharmacol. 161, 241-270. doi: 10.1111/j.1476-5381.2010. 00815.x

Whyte, M. P. (2010). Physiological role of alkaline phosphatase explored in hypophosphatasia. Ann. N.Y. Acad. Sci. 1192, 190-200. doi: 10.1111/j.17496632.2010.05387.x

Conflict of Interest Statement: The author declares that the research was conducted in the absence of any commercial or financial relationships that could be construed as a potential conflict of interest.

Received: 18 June 2014; accepted: 23 June 2014; published online: 11 July 2014.

Citation: Mansell JP (2014) Convergence of vitamin $D$ and lysophosphatidic acid signaling in stimulating human osteoblast maturation. Front. Physiol. 5:263. doi: $10.3389 /$ fphys.2014.00263

This article was submitted to Integrative Physiology, a section of the journal Frontiers in Physiology.

Copyright (c) 2014 Mansell. This is an open-access article distributed under the terms of the Creative Commons Attribution License (CC BY). The use, distribution or reproduction in other forums is permitted, provided the original author(s) or licensor are credited and that the original publication in this journal is cited, in accordance with accepted academic practice. No use, distribution or reproduction is permitted which does not comply with these terms. 